how a single experiment with photoelectric absorption optics can yield both partial specific volume and molecular weight. The former quantity is required for all ultracentrifugal molecular weight procedures. Its independent measurement requires a substantial amount of material, and solutions of precisely known dry-weight concentration. For proteins, a moderately reliable value can be calculated if the amino-acid composition is known and if there are no prosthetic groups; in many cases, however, and especially perhaps for nucleic acids, the partial specific volume is arguably the limiting factor in molecular weight determinations.

Edelstein and Schachman measure in a single experiment the sedimentation equilibrium distribution in $\mathrm{H}_{2} \mathrm{O}$ and $\mathrm{D}_{2} \mathrm{O}$ solutions of identical concentration in separate cells. They show that a correction, which is effectively the same for all proteins, can be applied to account for the small change in molecular weight resulting from hydrogen-deuterium exchange. From the standard equilibrium plots (ln $c$ against $r^{2}$ ) for the two solutions, the partial specific volume is easily derived, and an accuracy of 1 per cent is reported. The precision of the method can be further enhanced if $\mathrm{D}_{2}{ }^{18} \mathrm{O}$ is available as a solvent; for a typical protein the slope of the equilibrium plot is then nearly halved compared with $\mathrm{H}_{2} \mathrm{O}$ solution. Values of the partial specific volume and molecular weight obtained by these procedures for adenosine and a range of proteins are in remarkable agreement with accepted data. Edelstein and Schachman point out that their method can be applied even in the presence of other sedimenting species if the substance under study happens to have an absorption band in a separate region.

In referring to the work of Simon and Konigsberg (Proc. Nat. Acad. Sci., U.S., 56, 749; 1966) in this column on December 10, 1966, their conclusions were incorrectly represented. Whereas the first report by these authors (Fed. Proc., 25, 648; 1966) stated that cross-linked haemoglobins had been prepared which were incapable of dissociating into sub-units, the later article instead reports intramolecular cross-linking, and no bonds between sub-units. The annihilation of the haem-haem interactions evidently results therefore from the chemical disturbance of the $\beta$-chains as such and not from inhibition of sub-unit exchange.

\section{Survival of Starved Bacteria}

\section{by a Correspondent in Microbiology}

ORGANISMS might be expected to respond to adverse environments by a suspension of synthetic activities so as to become more independent of their surroundings but, in practice, bacteria rarely respond in this way. The synthesis of cell components may continue, possibly at altered rates, during prolonged starvation. Continued synthesis under starvation conditions leads to aberrant protoplasm which may ultimately prove lethal. In the current number of the Journal of Bacteriology are published a series of papers by Kennell, Kotoulas and Marchesi (J. Bact., 93(1), 334, 345 and 357 ; 1967) which discuss various aspects of nucleic acid and protein metabolism in starved Aerobacter aerogenes cultures. Starvation conditions were established by harvesting exponentially growing cells and resuspending them in the growth medium lacking magnesium. Although natural environments are probably rarely or never deficient in $\mathrm{Mg}^{++}$, this starvation system was studied because on present evidence magnesium is the most frequent metal co-factor for metallo-enzymes and is intimately concerned with ribosome stability.

In the $\mathrm{Mg}^{++}$deficient medium, $A$. aerogenes continued synthesizing nucleic acids and proteins for more than 70 hours provided that the source of carbon was not limiting. During the initial 24 hour period, the DNA content of the culture increased ten-fold, but the viable count showed only a three-fold increment during the first few hours and thereafter remained approximately constant. The ribosome population became "frozen" when the cells were incubated in the starvation medium. This constancy was maintained by the rate of ribosome degradation (induced by $\mathrm{Mg}^{++}$ deficiency) being paralleled by an equivalent rate of synthesis. Ribosome degradation was sequentially ordered and particles derived from degradation did not appear to be exchangeable with those in the process of assembly. Ribosomal proteins which had been synthesized during the preceding phase of growth in the complete medium were lost steadily during starvation and accounted for the total protein loss from the starving cells. Magnesium starvation of uracil-requiring strains of $A$. aerogenes produced confirmatory results: during uracil starvation a smaller fraction of the synthesized proteins were ribosomal and the amount of protein which decayed during the subsequent $\mathrm{Mg}^{++}$starvation was proportionally reduced.

Cytochemical changes accompanying $\mathrm{Mg}^{++}$starvation are also described by these authors (J. Bact., 93(1), 367; 1967). A progressive decrease in cell size occurred during the initial period when an expanding viable population was paralleling DNA synthesis. However, the subsequent phase of continued DNA synthesis and constant viable population showed cells becoming elongated into filamentous morphologies and, in addition, a second population of very small cells appeared. The latter, probably the result of disparate cell division, comprised an increasing proportion of the total population but (i) were unable to grow or divide when returned to complete media, and (ii) were devoid of biosynthetic activity as assessed by uracil- $\mathrm{H}^{3}$ incorporation into nucleic acids. Furthermore, these cells appeared almost completely lacking in ribosomes. After 20 hours of starvation, the very small cells accounted for more than 80 per cent of the population and were enough to explain the disparity between the total DNA content and the viable complement of the starved culture.

A continuation of normal cell division throughout starvation would lead to a diluting out of the ribosomes in the population so that the number per cell would be insufficient to maintain the metabolic potential and viability of the population. Kennell and his colleagues conclude that the very small cells arising from this apparently inevitable division activity are sacrificed in order to preserve a line of viable cellsthe filamentous cells. The regulation of such a differential survival mechanism is an intriguing problem and it remains to be seen whether this type of differentiation is general in starving bacteria under different starvation conditions. 